Cahiers de la recherche sur les droits

Cahiers

Fur les Droits fondamentaux

$8 \mid 2010$

La liberté d'expression

\title{
Racisme aux États-Unis : le premier amendement au secours de l'intolérance, pas de la violence
}

Amélie Robitaille-Froidure

\section{(2) OpenEdition}

Édition électronique

URL : https://journals.openedition.org/crdf/6157

DOI : $10.4000 /$ crdf.6157

ISSN : 2264-1246

Éditeur

Presses universitaires de Caen

Édition imprimée

Date de publication : 20 décembre 2010

Pagination : 83-90

ISBN : 978-2-84133-367-7

ISSN : $1634-8842$

\section{Référence électronique}

Amélie Robitaille-Froidure, «Racisme aux États-Unis : le premier amendement au secours de

l'intolérance, pas de la violence », Cahiers de la recherche sur les droits fondamentaux [En ligne], 8 | 2010, mis en ligne le 08 octobre 2020, consulté le 14 novembre 2022. URL : http://journals.openedition.org/ crdf/6157 ; DOl : https://doi.org/10.4000/crdf.6157 


\title{
Racisme aux États-Unis : le premier amendement au secours de l'intolérance, pas de la violence
}

\author{
Amélie ROBITAILLE-FROIDURE \\ Allocataire-moniteur à I'Université de Paris-Ouest Nanterre - La Défense \\ Centre de recherches et d'études sur les droits fondamentaux (CREDOF)
}

I. Le " paradoxe de la tolérance ": l'impossible prohibition du discours raciste

A. L'absence certaine de prohibition du discours raciste stricto sensu

B. L'absence probable de prohibition du discours négationniste

II. Tolérer les paroles mais pas les actes:

la prohibition du discours susceptible de provoquer la violence raciale

A. La prohibition certaine du discours incitant l'auditoire à commettre des actes racistes

B. La prohibition probable du discours adressé à une minorité raciale en vue de provoquer une réaction violente de sa part

Pierre angulaire de la démocratie pour les uns ${ }^{1}$, liberté matricielle pour les autres ${ }^{2}$, la liberté d'expression ne saurait toutefois permettre de dire ou d'écrire tout et n'importe quoi. À cet égard, nombre de conventions internationales et de législations internes viennent définir les limites de cette liberté et encadrer son exercice. C'est notamment le cas en ce qui concerne le discours raciste.

Sur le plan international, le racisme est réprimé, entre autres, par la Convention internationale sur l'élimination de toutes les formes de discrimination raciale ${ }^{3}$ et par le Pacte international sur les droits civils et politiques ${ }^{4}$. Sur le plan interne, l'exemple français est tout à fait caractéristique de la volonté étatique d'endiguer le discours raciste : sont prohibés l'injure et la diffamation raciales ${ }^{5}$, le discours négationniste ${ }^{6}$ et la provocation à la discrimination, à la haine et à la violence raciales ${ }^{7}$. Bien qu'elles aient parfois fait couler beaucoup d'encre, ces législations ne soulèvent pas de problème de constitutionnalité criant ${ }^{8}$.

1. Rapport du 30 novembre 1993 de la Commission européenne des Droits de l'homme dans l'affaire Vogt c. Allemagne, $\$ 71$, cité par F. Sudre et al., Les grands arrêts de la Cour européenne des Droits de l'homme, Paris, PUF, 2007, p. 580. Par ailleurs, «la liberté d'expression, pierre angulaire de la démocratie » a été le thème retenu par l'Agence de l'Union européenne pour les droits fondamentaux pour la conférence sur les droits fondamentaux dédiée à la problématique de la liberté d'expression les 8 et 9 décembre 2008.

2. B. Mathieu, «La liberté d'expression en France: de la protection constitutionnelle aux menaces législatives », Revue du droit public, $\mathrm{n}^{\circ} 1,1^{\mathrm{er}}$ janvier $2007, \mathrm{p}$. 231.

3. Article 4 de la Convention sur l'élimination de toutes les formes de discrimination raciale.

4. Article $20 \$ 2$ du Pacte international sur les droits civils et politiques.

5. Articles 32 alinéa 2, et 33 alinéa 3 de la loi du 29 juillet 1881. Voir JO du 30 juillet 1881, p. 4201.

6. Article 24 bis de la loi du 29 juillet 1881 inséré par la loi Gayssot nº 90-615 du 13 juillet 1990.

7. Article 24 alinéa 6 de la loi du 29 juillet 1881.

8. Certains auteurs réfutent cependant la constitutionnalité de la loi Gayssot - qui n’a pas été déférée au Conseil constitutionnel. Sur ce point, voir notamment J.-P. Feldman, «Peut-on dire n'importe quoi sur la Shoah ? », Revue de droit international et de droit comparé, n 4, 1998, p. 229-271 (267) ; M. Imbleau, 
La question se pose, en revanche, avec davantage d'acuité en ce qui concerne les États-Unis. En effet, le premier amendement à la Constitution américaine prévoit que «le Congrès ne fera aucune loi $[. .$.$] restreignant la$ liberté de parole ou de la presse ${ }^{9}$. Alors qu'une telle conception de la liberté d'expression ne laisse, a priori, pas de place à une éventuelle prohibition du discours raciste ${ }^{10}$, les États-Unis ont ratifié non seulement le Pacte international sur les droits civils et politiques mais également la Convention internationale sur l'élimination de toutes les formes de discrimination raciale. Qu'en conclure? Que la liberté d'expression ne connaît aucune limite aux États-Unis, ceci au mépris des engagements internationaux de ce pays? Que les obligations conventionnelles américaines entraînent une interprétation contra legem du premier amendement à la Constitution américaine adopté, rappelons-le, il y a plus de deux cents ans?

Seule une solution nuancée, pondérée, permet de résoudre un tel conflit. C'est sur cette voie que se sont engagés les États-Unis. En effet, il ne règne pas dans ce pays, contrairement à ce qui est parfois avancé, une conception «absolutiste» de la liberté d'expression : la Cour suprême a interprété le premier amendement de telle sorte que certaines limites - certes encadrées et circonscrites puissent y être apportées. C'est notamment le cas en ce qui concerne les publications des groupements prônant le renversement du Gouvernement ${ }^{11}$, ainsi que les publications obscènes ${ }^{12}$ ou pédophiles ${ }^{13}$. La ratification de conventions internationales prohibant le discours raciste semble donc aller dans le sens dans lequel la Cour suprême a interprété le premier amendement. Il serait toutefois hâtif d'en conclure que le discours raciste est prohibé en toutes circonstances outre-Atlantique. En effet, les États-Unis ont assorti la ratification de ces conventions de scrupuleuses réserves et déclarations interprétatives. Pour la délégation américaine, le Pacte de 1966 «n'autorise pas les États-Unis et n'exige pas d'eux qu'ils adoptent des lois ou autres mesures de nature à restreindre la liberté d'expression et d'association protégée par la Constitution et les lois des États-Unis ${ }^{14}$.

Le même type de réserve, rappelant l'importance de la liberté d'expression et vidant de leur sens les obligations conventionnelles en la matière, a été formulé lors de la ratification de la Convention internationale sur l'élimination de toutes les formes de discrimination raciale $^{15}$.

La position américaine est donc claire: les États-Unis n'entendent pas se voir imposer l'obligation de limiter la liberté d'expression, quand bien même cela se ferait au nom de la lutte contre le racisme. Ainsi, bien que les ÉtatsUnis aient ratifié les deux principales conventions internationales prohibant le discours raciste, force est de constater que les réserves et déclarations interprétatives émises par la délégation américaine assurent une large primauté à la liberté d'expression sur le droit à être protégé contre le racisme. C'est finalement en distinguant le discours raciste (I) de la provocation à la violence raciale (II) que la Cour suprême est parvenue à concilier le premier amendement avec les engagements internationaux souscrits par les États-Unis en matière de lutte contre le racisme.

\section{Le " paradoxe de la tolérance ${ }^{16}$ : l'impossible prohibition du discours raciste}

On assiste aujourd'hui à une évolution du phénomène raciste: partant, à l'origine, d'une théorie fondée sur la supériorité raciale, le racisme tend, à présent, à exprimer le refus de l'assimilation et du mélange ${ }^{17}$. Cette évolution aboutit à une redéfinition plus large: nous entendrons donc ici par «discours raciste» non seulement le discours raciste «traditionnel» fondé sur une prétendue hiérarchie des «races» mais aussi le racisme dans son acception plus englobante selon laquelle ce terme

La négation du génocide nazi, liberté d'expression ou crime raciste? Le négationnisme de la Shoah en droit international et en comparé, Paris, L'Harmattan, 2003, p. 261; M. Troper, "La loi Gayssot et la Constitution », Annales. Histoires et Sciences sociales, 54 (6), novembre-décembre 1999, p. 1239-1255; B. Beignier, "De la langue perfide délivre-moi. Réflexions sur la loi du 13 juillet 1990 dite "loi Gayssot" ", in Pouvoir et Liberté. Études offertes à J. Mourgeon, Bruxelles, Bruylant, 1998, p. 497-533 et B. Mathieu, «La liberté d'expression en France... », p. 231.

9. Traduction proposée par D. Colas, Textes constitutionnels français et étrangers, Paris, Larousse, 1994, p. 186. On signalera ici que toutes les traductions sont personnelles, sauf mention contraire.

10. La question de la prohibition du discours raciste a été soulevée pour la première fois dans les années 1930. En 1934, l'American Civil Liberty Union (ACLU) a publié un pamphlet intitulé Shall We Defend Free Speech for Nazis in America? dans lequel l'association répond à cette question par l'affirmative. Sur ce point, voir S. Walker, Hate speech: the history of an american controversy, Lincoln, University of Nebraska Press, 1994, p. 42.

11. Dennis v. United States, 341 US 494 (1951).

12. Miller v. California, 413 US 15 (1973).

13. New York v. Ferber, 458 US 747 (1982).

14. Réserve américaine au Pacte international sur les droits civils et politiques. La délégation américaine a ajouté dans une déclaration que « [...] 2) de l'avis des États-Unis, les États parties au Pacte doivent, dans la mesure du possible, s'abstenir d'imposer toutes restrictions ou limitations à l'exercice des droits consacrés et protégés par le Pacte, même lorsque ces restrictions et limitations sont permises aux termes de celui-ci».

15. Réserve américaine à la Convention internationale sur toutes les formes de discrimination raciale : « [...] 1) La Constitution et les lois des États-Unis prévoient des garanties étendues en faveur de la liberté de parole, d'expression et d'association des individus. En conséquence, les États-Unis n'acceptent aucune obligation en vertu de la présente Convention, en particulier ses articles 4 et 7, de nature à restreindre ces droits par l'adoption d'une législation ou de toute autre mesure, pour autant que ces derniers sont protégés par la Constitution et les lois des États-Unis ».

16. M. Rosenfeld, «La philosophie de la liberté d'expression en Amérique : la liberté d'expression en théorie et en pratique ", in L'Architecture du droit. Mélanges en l'honneur de Michel Troper, D. de Béchillon, P. Brunet, V. Champeil-Desplats et al. (dir.), Paris, Economica, 2006, p. 883-895 (892).

17. En ce sens, voir notamment P.-A. Taguieff, «Définition et évolution du phénomène raciste », in La Lutte contre le racisme et la xénophobie, Rapport de 1991, Commission nationale consultative des Droits de l'homme (éd.), Paris, La Documentation française, 1992, cité par F. Massias, «La liberté d'expression et le discours raciste ou révisionniste», Revue trimestrielle des Droits de l'homme, nº 13, 1993, p. 183-210 (184). 
désigne « la croyance qu'un motif tel que la race ${ }^{18}$, la couleur, la langue, la religion, la nationalité ou l'origine nationale ou ethnique justifie le mépris envers une personne ou un groupe de personnes ou l'idée de supériorité d'une personne ou d'un groupe de personnes $"{ }^{19}$.

Ce « rejet de l'autre» caractérise ce que nous qualifierons ici de racisme au sens strict. Une étude globale de ce phénomène ne saurait par ailleurs éluder l'analyse du négationnisme $e^{20}$ puisque celui-ci « constitue - pour paraphraser Clausewitz - la poursuite du racisme par d'autres moyens ${ }^{21}$.

La Cour suprême des États-Unis refuse que le Congrès - ou les États fédérés - viennent prohiber le discours raciste car «il existe un principe intangible qui fonde le premier amendement: le Gouvernement ne peut pas interdire l'expression d'une idée pour la seule raison que la société trouve cette idée en elle-même injurieuse ou désagréable ${ }^{22}$. Ce principe a d'autant plus de répercussions que la notion de symbolic speech a conduit la Cour suprême à appliquer le premier amendement non seulement aux paroles racistes mais également au message raciste que peut véhiculer un comportement (une manifestation, un incendie de croix, etc.). En effet, tant le discours raciste stricto sensu (A) que le négationnisme (B) sont couverts par la protection du premier amendement.

\section{A. L'absence certaine de prohibition du discours raciste stricto sensu}

S'il est aujourd'hui largement admis que le discours raciste est couvert par la protection du premier amendement, la Cour suprême a fait preuve de quelques hésitations en la matière ${ }^{23}$. Il a fallu attendre la fin des années 1970 et l'affaire Skokie ${ }^{24}$ pour que la Cour suprême se prononce contre la prohibition du discours raciste. En l'espèce, la Cour a conclu à l'inconstitutionnalité de l'ordonnance prise à l'encontre du parti national-socialiste interdisant à ses membres de défiler dans la commune de Skokie vêtus d'uniformes militaires et arborant des croix gammées ${ }^{25}$. Bien que les juges se soient « réfugi[és] derrière des motivations techniques liées aux conditions d'admission du sursis à exécution ${ }^{26}$, il n'en demeure pas moins qu'une telle manifestation est possible aux États-Unis. En effet, l'année suivante, la Cour suprême a refusé d'accorder un writ of certiorari pour se prononcer sur la constitutionnalité de plusieurs arrêtés adoptés par la commune de Skokie en vue d'interdire la venue du parti national-socialiste ${ }^{27}$. Ce faisant, la Cour suprême a confirmé la décision de cour du $7^{\mathrm{e}}$ circuit qui, après avoir constaté que les symboles nazis ne constituaient pas des fighting words ${ }^{28}$, avait retenu la violation du premier

18. L'ECRI (Commission européenne contre le racisme et l'intolérance) prend le soin de préciser que «tous les êtres humains appartenant à la même espèce, l'ECRI rejette les théories fondées sur l'existence de "races" différentes. Cependant, afin d'éviter de laisser sans protection juridique les personnes qui sont généralement et erronément perçues comme appartenant à une "autre race", l'ECRI utilise ce terme dans la présente recommandation ". Commission européenne contre le racisme et l'intolérance, Lutter contre le racisme tout en respectant la liberté d'expression, Actes du séminaire d'experts des 16 et 17 novembre 2006, Strasbourg, Conseil de l'Europe, 2007:

www.coe.int/t/dghl/monitoring/ecri/activities/22-freedom_of_expression_Seminar_2006/NSBR2006_proceedings_fr.pdf.

19. Recommandation de politique générale $\mathrm{n}^{\circ} 7$ de l'ECRI sur la législation nationale pour lutter contre le racisme et la discrimination raciale.

20. Alors que le révisionnisme « concerne la remise en cause de toute doctrine orthodoxe» (définition proposée par G. Cohen-Jonathan, « Négationnisme et Droits de l'homme ", Revue trimestrielle des Droits de l'homme, ${ }^{\circ} 32,1997, \mathrm{p} .571$ ), le négationnisme renvoie précisément à la négation du génocide juif et de l'existence des chambres à gaz durant la seconde guerre mondiale.

21. G. Haarscher, «Liberté d'expression, blasphème, racisme : essai d'analyse philosophique et comparée », Working Papers du Centre Perelman de philosophie du droit, $\mathrm{n}^{\circ}$ 2007/1, mis en ligne le 24 juin 2007, www.philodroit.be, p. 29.

22. Texas v. Johnson, 109 US 2533 (1989), traduction proposée par K. Bird, «L'impossible réglementation des propos à caractère raciste aux États-Unis ", Revue française de droit constitutionnel, 46, 2001, p. 265-287 (276).

23. Sur l'évolution de la jurisprudence américaine en la matière, voir J. Bell, « Restraining the heartless : racist speech and minority rights », 84 Ind. L. J. 963 (2009) - et sa traduction française : J. Bell, «Pour faire barrage à ceux qui n'ont pas de cœur: expressions racistes et droit des minorités », in $L a$ Liberté d'expression aux États-Unis et en Europe, E. Zoller (dir.), Paris, Dalloz, 2008, p. 51-69. Sur la question de la prohibition du discours raciste, il convient tout particulièrement de mentionner l'affaire Beauharnais (Beauharnais v. Illinois, 343 US 250 (1952)). En l'espèce, la Cour était saisie d'une loi de l'Illinois interdisant toute publication dépeignant «la dépravation, la criminalité, le manque de chasteté ou de vertu d'une catégorie de citoyens, de toute race, couleur ou religion [ou exposant] les citoyens de toute race, couleur ou religion, au mépris, à la dérision ou à l'opprobre ou [produisant] des troubles à l'ordre public ou des émeutes » (traduction proposée par L. Grosclaude, La Liberté d'expression dans la jurisprudence constitutionnelle de la Cour suprême des États-Unis, Thèse de doctorat soutenue en 2003 à l'Université du Panthéon Assas, E. Zoller (dir.), p. 178). Se référant à la notion de fighting words (Chaplinsky v. New Hampshire, 315 US 568 (1942)), la Cour suprême a jugé la loi de l'Illinois constitutionnelle considérant que si la diffamation individuelle (private libel) n'est pas protégée par le premier amendement, la diffamation collective (group libel) ne doit pas l'être non plus. Si, dans l'affaire Beauharnais, la Cour suprême a semblé accepter la prohibition du discours raciste, la notion de diffamation collective n'a pas été invoquée depuis lors et la juridiction suprême estime aujourd'hui que la diffamation individuelle est protégée par le premier amendement (New York Times Co v. Sullivan, 376 US 254 (1964)). C'est pourquoi de nombreux auteurs avancent que la notion de diffamation collective a implicitement été abandonnée par les juges américains (en ce sens, voir notamment F. Haiman, «Speech and law in a free society» [1981], cité par D. Kretzmer, «Freedom of speech and racism ", Cardozo Law Review, vol. 8, 1987, p. 445 et L. Tribe, American Constitutional Law, Mineola - New York, Foundation Press, 1988, cité par R. Delgado, "Campus antiracism rules : constitutional narratives in collision », Northwestern University Law Review, vol. 85, 1991 , p. 343.

24. Pour un résumé des faits de l'affaire Skokie, voir L. Grosclaude, La Liberté d'expression dans la jurisprudence constitutionnelle..., p. $691-696$ et S. Walker, Hate speech..., p. 120-126. Pour un exposé plus précis, voir P. Strum, When the nazis came to Skokie: freedom of speech we hate, Lawrence, University Press of Kansas, 1999.

25. National Socialist Party v. Skokie, 432 US 43 (1977).

26. L. Pech, La Liberté d'expression et sa limitation: les enseignements de l'expérience américaine au regard d'expériences européennes (Allemagne, France et Convention européenne des Droits de l'homme), Thèse de doctorat soutenue en 2001 à l'Université d'Aix Marseille III, G. Scoffoni (dir.), ClermontFerrand - Paris, Presses universitaires de la Faculté de droit - LGDJ, 2003, p. 385.

27. Smith v. Collin, 436 US 953 (1978).

28. Il s'agit des «mots qui infligent un dommage». En ce sens, voir S. Walker, Hate speech..., p. 105 («words that by their very utterance inflict injury»). 
amendement. Bien que la Cour suprême ne se soit pas explicitement prononcée sur la question de la prohibition du discours raciste dans l'affaire Skokie, il est apparu clairement que, pour les juges américains, de telles mesures sont contraires au premier amendement.

C'est la solution qu'a finalement retenue la Cour suprême en 1992 dans l'affaire RAV v. City of Saint Paul. La disposition contestée prévoyait que « quiconque place sur une propriété publique ou privée, un symbole, un objet, une injure, un caractère ou un graffiti, incluant, notamment mais pas seulement l'incendie d'une croix ou d'une croix gammée, tout en sachant ou en ayant des raisons raisonnables de savoir que cela provoquera la colère, la peur ou l'indignation d'un individu en considération de sa race, de sa couleur, de ses croyances [...] commet une conduite contraire aux bonnes mœurs et se rend coupable d'un délit ${ }^{29}$.

La Cour a conclu à l'inconstitutionnalité de cette disposition en ce qu'elle visait « toute forme d'injures en général ${ }^{30}$ mais a toutefois jugé nécessaire de préciser: «qu'il n'y ait pas d'erreur, nous pensons que brûler une croix dans la cour de quelqu'un est répréhensible. Mais Saint Paul a suffisamment de moyens à sa disposition pour empêcher de tels comportements, sans jeter le premier amendement dans le brasier $»^{31}$.

Ainsi, le juge américain considère aujourd'hui que le discours raciste est protégé par le premier amendement ${ }^{32}$. Après les hésitations dont elle a fait preuve et face aux - quelques - critiques doctrinales ${ }^{33}$, la Cour suprême n'a eu d'autres choix que de justifier largement sa position.

Le refus de prohiber le discours raciste repose, entre autres, sur la distinction entre les faits et les opinions. Pour le juge américain, si les opinions sont largement protégées par le premier amendement, les faits ne le sont pas dès lors qu'il est possible d'établir leur existence avec certitude. Selon cette distinction, le discours raciste est considéré comme une opinion. Il est donc couvert par la protection du premier amendement. Même si, comme le souligne R. Smolla, une telle distinction peut être remise en cause - ce qui rendrait la prohibition du discours raciste conforme à la Constitution ${ }^{34}$-, les juges et la majorité des auteurs américains continuent à s'y référer pour justifier la prohibition du discours raciste.

Par ailleurs, au fil des affaires ayant trait à la liberté d'expression dont elle a été saisie, la Cour suprême a élaboré deux théories qui lui ont permis de développer une jurisprudence très libérale en la matière. Il s'agit des théories de la slippery slope et du chilling effect. Selon la théorie de la slippery slope, il est peu probable qu'une exception à la liberté d'expression demeure un cas isolé. Celle-ci risque, au contraire, d'être invoquée comme un précédent afin de justifier davantage de limitations. C'est pourquoi, il importe d'éviter toute entrave à la liberté d'expression, quelle que soit la nature du discours concerné. La Cour suprême - très soucieuse de ne pas s'engager sur cette «pente glissante» - rappelle fréquemment le principe selon lequel «le Gouvernement doit rester neutre dans le libre marché des idées ${ }^{35}$. La théorie du chilling effect a, pour sa part, été énoncée dès 1927 par la Cour suprême dans l'affaire Whitney ${ }^{36}$. La formule de M. Barringer "more speech, not less, is the proper cure for offensive speech ${ }^{37}$ résume parfaitement cette théorie selon laquelle toute limitation à la liberté d'expression et a fortiori la prohibition du discours raciste - vient «geler» la discussion. Le refus de prohiber le discours raciste reposerait donc sur la nécessité de maintenir le libre marché des idées, un tel discours pouvant «avoir des effets bénéfiques pour la majorité du public en forçant cette dernière à dépasser son conformisme, et à discuter des idées perturbatrices et dérangeantes ${ }^{38}$.

Bien qu'elle soit quelque peu contestée, la position des juges américains ne semble pas susceptible de subir un revirement de jurisprudence en matière de discours raciste stricto sensu. Elle est aujourd'hui largement motivée et bien établie. Ce qui n'est pas le cas concernant le discours négationniste.

29. St. Paul, Minn., Legis. Code $\$ 292.02$ (1990), cité par O. Fiss, «The Supreme Court and the problem of hate speech», Cap. U. Law Review, vol. 24, 1995, p. 281: "Whoever places, on public or private property a symbol, object, appellation, characterization or graffiti, including, but not limited to, a burning cross or Nazi swastika, which one knows or has reasonable grounds to know arouses anger, alarm or resentment in others on the basis of race [or] color [...] commits disorderly conduct and shall be guilty of misdemeanor».

30. J. Bell, « Pour faire barrage... », « Restraining the heartless... », p. 60 de la version française.

31. RAV v. City of Saint Paul, 505 US 377 (1992), traduction proposée par L. Grosclaude, La Liberté d'expression dans la jurisprudence constitutionnelle..., p. 700.

32. Il convient toutefois de noter que le discours raciste proféré par des agents publics - et notamment par des policiers - n'est pas protégé par le premier amendement. En ce sens, voir A. G. Travieso, «Employees free speech rights in the workplace : balancing the First Amendment against racist speech by police officers", Rutgers Law Review, vol. 51, 1999, p. 1377.

33. En ce sens, voir R. Delgado, "Words that wound: a tort action for racial insults, epithets and name-calling», Harvard Civil Rights-Civil Liberties Law Review, vol. 17, 1992, p. 133 ; C. R. Lawrence III, «If the hollers let him go : regulating racist speech on campus », Duke Law Journal, 1990, p. 431; M. J. Matsuda, "Public response to racist speech, considering the victim's story ", Michigan University Law Review, vol. 87, 1989, p. 2320, et R. A. Smolla, «Rethinking First Amendment assumption about racist and sexist speech», Washington and Lee University Law Review, vol. 47, 1990, p. 171.

34. R. A. Smolla, « Rethinking First Amendment assumption... », p. 179-198.

35. Hustler Magazing v. Falwell, 485 US 56 (1988) : «The government must remain neutral in the marketplace of ideas ». En l'espèce, la Cour cite l'arrêt FCC v. Pacifica Found, 438 US 726 (1978).

36. Withney v. California, 274 US 357 (1927) : «If there be time to expose through discussion the falsehood and fallacies, to avert the evil by the processes of education, the remedy to be applied is more speech, not enforced silence».

37. M. Barringer, «Campus battle pits freedom of speech against racial slurs », New York Times, 25 avril 1989, \$1, at.1, col.1, cité par R. Delgado, «Campus antiracism rules: constitutional narratives in collision ", Northwestern University Law Review, vol. 85, 1991, p. 343.

38. L. Bollinger, The Tolerant Society: Freedom of Speech and Extremist Speech in America, Oxford, Oxford University Press, 1986, cité par M. Rosenfeld, "La philosophie de la liberté d'expression en Amérique... », p. 892. 


\section{B. L'absence probable de prohibition du discours négationniste}

Malgré l'absence de décision de principe s'agissant de la prohibition du discours négationniste, la majorité des auteurs estime qu'il est plus que probable que la Cour suprême la considérerait incompatible avec le premier amendement ${ }^{39}$. C'est d'ailleurs la position qu'ont retenue les juges du fond qui, à plusieurs reprises, ont eu à se prononcer sur la question de la prohibition du discours négationniste.

Une décision de la Cour d'appel du $9^{\mathrm{e}}$ circuit $^{40}$ est caractéristique à cet égard. En l'espèce, $\mathrm{M}$. Mc Calden et l'Association des bibliothèques californiennes avaient conclu un contrat permettant à celui-ci de louer une salle en vue d'organiser une conférence intitulée «La liberté d'expression et l'Holocauste. Une analyse de la censure sévère et du terrorisme intellectuel qui empêchent toute recherche objective, tout débat ouvert sur ce sujet controversé». L'Association des bibliothèques californiennes ayant annulé le contrat après que le Comité juif américain et la municipalité l'ont promptement invitée à le faire, M. Mc Calden a saisi le juge. Celui-ci lui a donné raison considérant que, bien que le message qu'il entendait faire passer soit «répugnant ${ }^{41}$, l'annulation du contrat était illégale en ce qu'elle violait la liberté d'expression protégée par le premier amendement.

Il convient toutefois de préciser que si le négationnisme ne peut être prohibé en raison du message qu'il véhicule, il peut être réprimé «du moment que la sanction ne repose pas sur des considérations liées au contenu de l'opinion exprimée, quant à son caractère indigne ou faux, mais sur ses conséquences morales ou matérielles ${ }^{42}$. Ainsi, le juge américain admet qu'une revue universitaire refuse de publier un article négationniste ${ }^{43}$. Il estime également que l'organisation d'un concours offrant 50000 dollars de récompense à qui parviendra à prouver que les chambres à gaz nazies ont exterminé des juifs tombe sous le coup du délit de «préjudice émotionnel intentionnellement infligé » puisque « le fait que des juifs furent gazés à Auschwitz est incontestable» ${ }^{44}$.

En conséquence, bien que la Cour suprême ne se soit pas prononcée expressément sur la question de la prohibition du discours négationniste, de nombreuses décisions des juridictions ordinaires permettent de penser que si elle était saisie de telles dispositions, la juridiction suprême conclurait très probablement à leur non-conformité avec le premier amendement. Toutefois, en l'absence de décision de principe de la Cour suprême, les juges ordinaires et les auteurs américains ont été contraints de justifier abondamment l'inconstitutionnalité de la prohibition du discours négationniste.

La transposition des théories de la slippery slope et du chilling effect en la matière ne pose aucun problème en soi. En effet, on peut aisément comprendre que la prohibition du négationnisme risque d'entraîner les juges sur une «pente glissante » et de venir « geler » la discussion et la recherche. À cet égard, l'Association américaine des professeurs d'université a pris position contre la prohibition du discours raciste et négationniste sur les campus, considérant que la liberté d'expression est « la pré-condition de la recherche académique ${ }^{45}$. Si la transposition des théories de la slippery slope et du chilling effect à la prohibition du discours négationniste ne pose aucun problème, la transposition de la distinction entre les faits et les opinions s'avère, quant à elle, pour le moins problématique. En effet, l'Holocauste constitue indéniablement un fait et non pas une opinion. Ainsi, selon la classification traditionnelle opérée entre les faits et les opinions, le discours négationniste pourrait, en principe, être prohibé ${ }^{46}$. Conscientes de ce que la transposition de la distinction entre les faits et les opinions est inopérante s'agissant du discours négationniste, les juridictions ordinaires ont préféré, à notre connaissance, éluder cette question. C'est très probablement aussi pour cette raison que la doctrine américaine a tenté d'apporter d'autres arguments justifiant l'interdiction de prohiber le discours négationniste ${ }^{47}$.

39. En ce sens, voir G. Haarscher, «Liberté d'expression, blasphème, racisme... », p. 30 et A. Lewis, Freedom for the thought that we hate, New York, Basic Books, 2007, p. 158.

40. Mc Calden v. California Library Ass'n, 955 F.2d 1214 (1990).

41. Ibid., opinion dissidente commune: «No one disputes Mc Calden's right to say his piece, repugnant though his message be. The federal courts have a long and proud tradition of protecting the rights of individuals with unpopular points of view to express themselves even where this subjects onlookers to intense discomfort, even anger».

42. L. Pech, «Conflits entre différentes conceptions de la liberté d'expression sur Internet : vers l'imposition d'une lex americana en matière de lutte contre le discours raciste et négationniste? ", Légipresse, $\mathrm{n}^{\circ}$ 188, II, 2002, p. 5-10 (9).

43. Sur ce point, voir K. Lasson, "Holocaust denial and the First Amendment: the quest for truth in a free society », Geo. Mason. Law Review, vol. 6, 35, 1997 , p. 58-60.

44. Mermelstein v. Institute for Historical review, $\mathrm{n}^{\circ} \mathrm{C} 356542$ (Cal. Super. Ct. July 22, 1985): «[...] the fact that Jews were gassed at Auschwitz is indisputable».

45. S. Walker, Hate speech..., p. 136: "[ free speech is] the very precondition of the academic enterprise itself».

46. Le discours révisionniste pourrait, en revanche, éventuellement être considéré comme une opinion : ainsi, la relativisation du nombre de victimes de l'Holocauste serait protégée par le premier amendement.

47. K. Lasson se réfere à T. Jefferson pour qui « [...] la meilleure façon de découvrir les erreurs est de permettre leur correction par la vérité» (K. Lasson, "Holocaust denial and the First Amendment...", p. $53:$ : [...] the best way to deal with error is to permit its correction by truth»). E. Barendt souligne, quant à lui, le fait que la prohibition du discours négationniste risque de faire passer les auteurs de tels propos pour « des martyrs de la cause de la liberté d'expression » et d'accorder «plus de publicité» à leurs discours (E. Barendt, Freedom of speech, Oxford, Clarendon Press, 2005, p. 175: « [...] a prosecution and trial may [...] make the speaker or publisher a martyr to the cause of free speech» et p. $176:$ : [...] might have the unfortunate effect of giving its proponents and their claims more publicity than they would otherwise enjoy»). 
La prohibition du discours raciste et négationniste est donc inconcevable aux États-Unis. Il serait toutefois hâtif d'en conclure que les dispositions du Pacte international sur les droits civils et politiques et celles de la Convention sur l'élimination de toutes les formes de discrimination raciale ne sont pas respectées outre-Atlantique.

\section{Tolérer les paroles mais pas les actes: la prohibition du discours susceptible de provoquer la violence raciale}

La Cour suprême estime que la prohibition du discours susceptible d'entraîner la violence raciale est conforme aux prescriptions du premier amendement. Pour ce faire, les juges recourent à la théorie du clear and present danger qui, après avoir été énoncée dans des opinions dissidentes ${ }^{48}$, a été reprise par la Cour suprême ${ }^{49}$. La jurisprudence américaine admet donc que la liberté d'expression puisse être limitée dès lors que sont réunis les trois éléments permettant de caractériser un danger clair et imminent : «les circonstances doivent impliquer un mal substantiel extrêmement sérieux dont le degré d'imminence est très élevé ${ }^{50}$. Le standard du clear and present danger permet donc la mise en balance de la liberté d'expression et d'autres impératifs, tels que la lutte contre le racisme.

Alors que dès les années 1950, les références à la notion de clear and present danger se sont faites plus rares dans les arrêts de la Cour suprême ${ }^{51}$, le juge américain a néanmoins pris le parti d'appliquer cette théorie en matière de provocation. La provocation, qui désigne le «discours prononcé avec l'intention de provoquer une action $»^{52}$, est classée en deux catégories par les auteurs américains. D’une part, le discours par lequel «le locuteur entend inciter son auditoire à réaliser une action [violente] ${ }^{53}$. D'autre part, le discours par lequel «le locuteur entend faire réagir violemment à ses propos la personne à laquelle il s'adresse ${ }^{54}$. Ainsi, dans le cas du discours susceptible d'entraîner la violence raciale, il convient de distinguer le discours par lequel le locuteur entend inciter son auditeur à commettre un acte violent à l'égard d'une personne ou d'un groupe de personnes en raison de son appartenance - vraie ou supposée - à un groupe ethnique ou racial (A), du discours raciste adressé à une ou plusieurs personnes d'un tel groupe en vue de les faire réagir violemment $(\mathrm{B})$.

\section{A. La prohibition certaine du discours incitant l'auditoire à commettre des actes racistes}

La Cour suprême a admis très tôt et sans équivoque la constitutionnalité de la prohibition du discours par lequel un locuteur entend susciter une réaction raciste chez son auditoire. De telles (ré) actions sont qualifiées, aux ÉtatsUnis, de biased crimes, c'est-à-dire d' « infractions commises contre des individus intentionnellement choisis en raison de leur race ${ }^{55}$. Saisie de la condamnation d'un membre du Klu Klux Klan en application d'une loi de l'État d'Ohio réprimant l'apologie de la violence ou l'utilisation de moyens illégaux à des fins d'accomplir des réformes politiques, la Cour suprême a considéré qu'une telle loi n'est pas contraire aux prescriptions du premier amendement ${ }^{56}$.

La Cour se montre toutefois très exigeante en la matière : elle n'admet la prohibition de la provocation à la violence que si celle-ci produit un effet immédiat et elle prend en considération les autres moyens dont disposent l'État ou le Congrès pour l'empêcher. La provocation à la violence raciale est donc couverte par la protection du premier amendement «tant qu'elle ne franchit pas la ligne de démarcation entre le "plaidoyer" pour la violence et l' "incitation" à la violence ${ }^{57}$. Conscients de ce que ces deux notions sont très proches, les juges de la Cour suprême ont pris soin de justifier abondamment pourquoi seul le discours susceptible de provoquer la violence raciale peut se voir prohiber. Pour cela, ils se réfèrent à

48. Schenck v. United States, 249 US 47 (1919), opinion dissidente du juge Holmes: " The most stringent protection of free speech would not protect a man in falsely shouting fire in theatre and causing a panic [...]. The question in every case is whether the words are used in such circumstances and are of such a nature as to create a clear and present danger $»$. En ce sens, voir également Abrams v. United States 250 US 616 (1919), opinion dissidente des juges Holmes et Brandeis et Whitney v. California, 274 US 357 (1927), opinion dissidente du juge Brandeis.

49. Gitlow v. New York, 268 US 652 (1925): «In such case it has been held that the general provisions of the statute may be constitutionally applied to the specific utterance of the defendant if its natural tendency and probable effect was to bring about the substantive evil which the legislative body might prevent». La théorie du clear and present danger a finalement été théorisée dans l'affaire Bridges (Bridges v. California, 62 US 190 (1941)) avant d'être remaniée dans l'affaire Dennis (Dennis v. United States, 341 US 494 (1951)) : ce standard ne peut pas être «cristallisé en une règle rigide qui doit s'appliquer inflexiblement sans prêter attention aux circonstances de chaque affaire », traduction proposée par L. Pech., La Liberté d'expression et sa limitation..., p. 106.

50. J. Hemmer, Communication Law: the Supreme Court and the First Amendment, Lanham, University Press of America, 2006, p. $8:$ : [...] the Court stressed three elements that had to exist in order to find a "clear and present danger": 1) the circumstance must involve a "substantial evil", 2) the anticipated result must be "extremely serious", and 3) the "degree of imminence" of the danger must be extremely high" ".

51. En ce sens, voir J. Hemmer, Communication Law..., p. 8.

52. R. A. Smolla, «Rethinking First Amendment assumption... », p. 188-189: «[...] statements of incitement are statements made with the intent and form of inducing action $»$.

53. Ibid. : «[...] the speaker may seek to incite allies to help effectuate the speaker's purpose».

54. Ibid.: «[...] the speaker may seek to incite enemies to react against him».

55. K. Bird, «L'impossible réglementation... », p. 284.

56. Brandenburg v. Ohio, 395 US 444 (1969) : « [...] the constitutional guarantees of free speech and free press do not permit a State to forbid or proscribe advocacy of the use of force or of law violation except where such advocacy is directed to inciting or producing imminent lawless action and is likely to incite or produce such action».

57. M. Rosenfeld, «La philosophie de la liberté d'expression en Amérique... », p. 892. 
l'intention originelle des Pères fondateurs et recourent à la distinction entre les faits et les opinions.

Tout d'abord, le juge Holmes est venu justifier la théorie du clear and present danger en procédant à une interprétation historique du premier amendement: «ni Hamilton ni Madison, ni personne d'autre à cette époque ou plus tard, n'a jamais voulu que le discours tendant à conseiller au meurtre soit protégé par le premier amendement au nom de la liberté d'expression. ${ }^{58}$

En l'espèce, le recours à l'intention originelle des Pères fondateurs permet au juge d'asseoir et de légitimer une jurisprudence qui, pourtant, n'avait rien d'évident puisqu'il est en effet très délicat de distinguer le discours susceptible de provoquer la violence raciale du discours raciste stricto sensu. En se référant à l'intention originelle des Pères fondateurs, le juge Holmes invoque, en toute connaissance de cause, un argument quasi irréfragable.

Par ailleurs, la distinction entre les faits et les opinions permet également de justifier la prohibition du discours susceptible de provoquer la violence raciale ${ }^{59}$. Saisie de la constitutionnalité d'une loi érigeant en circonstance aggravante le fait de choisir intentionnellement sa victime en raison de son appartenance raciale ou ethnique (biased crimes), la Cour suprême a estimé qu’ «il est possible de prendre en compte le motif raciste d'un crime puisqu'un tel motif peut à juste titre être considéré comme une circonstance aggravante ${ }^{60}$. Ce faisant, les juges américains distinguent clairement les comportements des propos à caractère raciste. C'est bien parce qu'elle opère une distinction entre les faits et les opinions que la Cour suprême estime que la prohibition du discours susceptible de provoquer la violence raciale est conforme aux prescriptions du premier amendement.

Il ressort donc clairement de l'analyse de la jurisprudence américaine qu'il est possible de prohiber le discours susceptible de provoquer des crimes racistes de la part d'un auditoire. La question de la prohibition du discours raciste adressé à une minorité raciale ou ethnique en vue de provoquer une réaction violente de sa part, bien que probable, demeure quant à elle incertaine.

\section{B. La prohibition probable du discours adressé à une minorité raciale en vue de provoquer une réaction violente de sa part}

Sans avoir été explicitement admise par les juges américains, la prohibition du discours raciste adressé à une minorité raciale en vue de provoquer une réaction violente de sa part ne semble pourtant pas impossible. C'est du moins ce qui ressort de l'analyse non pas du dispositif mais des motifs de l'arrêt rendu en 2003 par la Cour suprême dans l'affaire Virginia v. Black ${ }^{61}$.

Certes, cet arrêt semble, de prime abord, exclure toute prohibition du discours raciste adressé à une minorité raciale en vue de provoquer une réaction violente de sa part: en l'espèce, la Cour suprême a considéré que la loi pénalisant le fait de brûler une croix (cross burning) avec l'intention d'intimider la victime (the intent to intimidate) n'est pas conforme au premier amendement. Pour le juge, malgré le message de "white supremacy» associé aux incendies de croix qui étaient traditionnellement le fait de membres du Klu Klux Klan, « il peut arriver que le fait de brûler une croix relève du discours politique, aussi répugnant en soit le contenu ${ }^{62}$. Ainsi, la prohibition du discours raciste adressé à une minorité raciale en vue de provoquer une réaction violente de sa part semble contraire aux exigences posées par le premier amendement.

Pour comprendre la brèche que pourrait ouvrir l'arrêt rendu dans l'affaire Virginia v. Black, il convient d'en examiner les motifs: dans son raisonnement, la Cour suprême semble sinon admettre, du moins ne pas exclure, que le discours adressé à une minorité raciale en vue de provoquer une réaction violente de sa part puisse se voir prohibé.

En effet, la Cour reprochait à la disposition de l'État de Virginie de ne pas opérer de distinction entre «un incendie de croix accompli dans le but de créer de la colère ou du ressentiment et celui accompli dans le but de menacer ou d'intimider une victime ${ }^{63}$. A contrario, on pourrait en conclure que si la disposition en cause s'était bornée à prohiber l'incendie de croix «accompli dans le but de créer de la colère ou du ressentiment», autrement dit celui susceptible de provoquer une réaction violente de la part de la personne visée, la Cour aurait estimé qu'elle était conforme au premier amendement.

Comme le souligne G. Haarscher, le juge américain exige que «le lien entre le discours et l'action soit court $»{ }^{64}$. Il est donc probable que si elle était saisie d'une loi prohibant uniquement le discours raciste adressé à une minorité raciale en vue de provoquer une réaction violente de sa part, la Cour suprême pourrait conclure à sa constitutionnalité. Bien évidemment, il ne s'agit là que d'une hypothèse d'école. En l'absence de décision de la juridiction suprême - et même, à notre connaissance, des

58. Frohwerk v. United States, 249 US 204 (1919), opinion dissidente du juge Holmes : « [...] we venture to believe that neither Hamilton nor Madison, nor any other competent person then or later, ever suppose that to make criminal the counselling of a murder within the jurisdiction of Congress would be an unconstitutional interference with free speech $»$.

59. Wisconsin v. Mitchell, 508 US 476 (1993).

60. I. Loveland, Importing the First Amendment: freedom of expression in American, English and European law, Evanston, Northwestern University Press, 1999, p. 159: «[...] evidence of racial animus towards the victim could be taken into account in determining whether to impose the death sentence for murder, because such animus could $[\ldots]$ legitimately be regarded as aggravating".

61. Virginia v. Black, 538 US 343 (2003). Pour un résumé des faits de cette affaire, voir notamment G. Haarscher, «Liberté d'expression, blasphème, racisme... », p. 22-23 et A. Tsesis, "Dignity and speech : the regulation of hate speech in a democracy", Wake Forest Law Review, vol. 44, 2009 , p. 497.

62. G. Haarscher, "Liberté d'expression, blasphème, racisme... ", p. 23-24.

63. Virginia v. Black, 538 US 343 (2003), traduction proposée par L. Grosclaude, La Liberté d'expression dans la jurisprudence constitutionnelle..., p. 706.

64. G. Haarscher, «Liberté d'expression, blasphème, racisme... », p. 24. 
juridictions inférieures - sur la question, il est impossible d'affirmer de manière péremptoire que la prohibition du discours raciste adressé à une minorité raciale en vue de provoquer une réaction violente de sa part est conforme avec le premier amendement.

Contrairement au discours raciste stricto sensu et au discours négationniste, le discours susceptible de provoquer la violence raciale n'est donc pas couvert par la protection du premier amendement. C'est cette distinction entre le racisme et la provocation à la violence raciale qui permet au juge américain de concilier les exigences du premier amendement et celles des engagements internationaux souscrits par les États-Unis en matière de lutte contre le racisme. Il convient néanmoins de préciser que la Convention internationale sur toutes les formes de discrimination raciale propose une définition plus large de la notion de provocation raciale que celle retenue par les juges américains : elle prévoit, en effet, que soient pro- hibées non seulement la provocation à la violence mais également la provocation à la discrimination et à la haine raciales. Certains auteurs américains proposent d'amender la Convention de telle sorte que seule la provocation à la discrimination ou à la violence raciales soit prohibée ${ }^{65}$. Une telle modification ne nous semble ni souhaitable ni nécessaire. En effet, il ne faut pas perdre de vue le fait que «lorsqu'il commence à se manifester par le verbe, le racisme ne tarde pas aussi à se manifester par les actes $"{ }^{66}$. Ainsi, l'absence de prohibition du discours susceptible de provoquer la haine raciale pourrait se révéler dangereuse. Il faut toutefois se garder de tomber dans l'excès inverse, à savoir la sur-réglementation. Au législateur français qui semble s'acheminer sur cette voie, il convient de rappeler l'aphorisme de Jean Rivero, selon lequel «la conciliation [...] se résume dans une formule si banale qu'elle relève du plus simple bon sens : réglementer, certes, mais réglementer moins, et réglementer mieux ${ }^{67}$.

65. M. Korengold, «Lessons in confronting racist speech : good intentions, bad results and article 4 (A) of the Convention on the elimination of all forms of racial discrimination ", Minnesota Law Review, vol. 77, 1993, p. 719: «[...] prohibition of incitement to racial discrimination or acts of violence».

66. Commission nationale consultative des Droits de l'homme, La Lutte contre le racisme et la xénophobie, Rapport 1994, Paris, La Documentation française, 1995, cité par J.-P. Feldman, « Peut-on dire n'importe quoi sur la Shoah? », p. 260.

67. J. Rivero, «Les limites de la liberté», in Libertés. Mélanges en l’honneur de Jacques Robert, Paris, Montchrestien, 1998 , p. 193. 